\title{
Application on Polynomial Approximation Method in Airborne Geological Exploration
}

\author{
Dawei Yin ${ }^{1}$, Jun $\operatorname{Lin}^{1}$ and Kaiguang $\mathrm{Zhu}^{1{ }^{1 *}}$ \\ ${ }^{1}$ College of Instrumentation \& Electrical Engineering, Jilin University, Changchun, 130021- China
}

Received 15 May 2013; Accepted 25 July 2013

\begin{abstract}
Coil motion noise is the main noise source in AEM data caused by the movement of the receiver coil in the Earth's magnetic field. The characteristics of both time domain and frequency domain of motion noise are analyzed. In this paper we present a scheme of removing motion noise which employs polynomial to represent motion noise for each half cycle. A set of equations are set up according to the characteristics of AEM data, and Lagrange method is employed to solve minimum problem with two constraint conditions together. The polynomial coefficients are calculated by matrix inverse and finally motion noise is subtracted from the AEM data half cycle by half cycle. Polynomial approximation method is applied to the synthetic data contaminated by low frequency motion noise comparing with traditional high pass filter method. The corrected AEM data shows polynomial approximation method is more effective in dealing with motion noise.
\end{abstract}

Keywords: Airborne Time Domain Electromagnetic Data, Coil Motion Noise, Polynomial Approximation, High Pass Filter

\section{Introduction}

AEM (Airborne electromagnetic method) is a universal kind of airborne geological exploration method, and has a series of advantages, such as rapid exploration velocity, lower cost, wide exploration range, and use in the sea field. Fig.1 shows airborne geological exploration course by helicopter. In China, geological exploration is faced with many difficulties because of complex terrain and numerous mountainous areas. Therefore, AEM is worth widespread application as a convenient exploration tool. With airborne electromagnetic data gathering, noise mixed in airborne electromagnetic data must be considered and which directly influences effective time window of airborne electromagnetic data and data inversion, especially influences reliability of late time data reflecting the Earth's depth information.

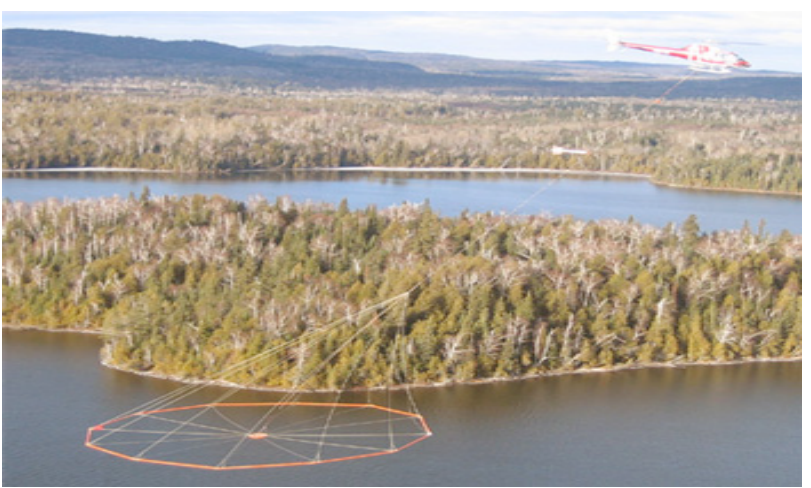

Fig. 1. Airborne geological exploration course by helicopter

\footnotetext{
*E-mail address: effortman0434@163.com

ISSN: 1791-2377 @ 2013 Kavala Institute of Technology. All rights reserved.
}

In recent years, with rapid development of computer and signal processing technology, a various kind of signal denoising methods in airborne electromagnetic data are developed by scholars at home and abroad[1,2]. For example, Sefric noise was reduced by pruning (Macnae, 1984), non-linear filtering (Lane, 1998), robust stastical method (Buselli, 1996), prediction of neural network according to ground sferic recording reference (Buselli, 1998), and wavelet transform (Lane 2000; Ridsdill 1999) $[3,4,5,6,7]$.

Coil motion noise is the main noise source in airborne electromagnetic exploration, and which is caused by induced electromotive force from flux variation of receiver coil cutting magnetic force lines of geomagnetic field in flight. Average intensity of geomagnetic field is $6 \times 10^{-5} \mathrm{~T}$. if the receiver coil turns 10 degree of yawing per second, assuming an effective area of $2000 \mathrm{~m}^{2}$ (including front amplifier, real area, turns), the effective area will decrease $232.8 \mathrm{~m}^{2}$, and then $14 \mathrm{mV}$ induced voltage will be produced in the receiver coil. Lane (1998, Fig.2) gave the coil motion noise profile at high altitude of $300 \mathrm{~m}$, with the amplitude of $10-30 \mathrm{mV}$, recorded by a fixed-wing time domain system QUESTEM without any transmitting current. QUESTEM is a fixed-wing time domain system operating with an alternating half-sine transmitting pulse width of $4 \mathrm{~ms}$ at a base frequency of $25 \mathrm{~Hz}$. The effective area of QUESTEM receiver coil is of $17,400 \mathrm{~m}^{2}$. Thus the normalized induced voltage from coil motion noise at high altitude is from $574 n T / s$ to $2298 n T / s$. The amplitude of coil motion noise recorded at survey altitude can be up to an order of 
magnitude higher than that recorded at high altitude under straight and level flight and calm air conditions because of near-ground turbulence and the pilot's efforts to maintain constant terrain clearance (Lane, 1998).

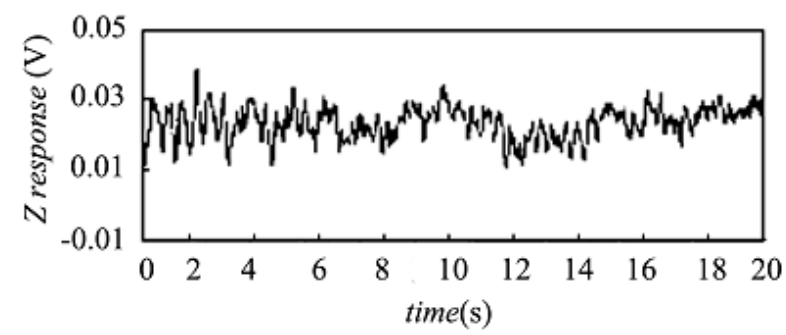

Fig. 2. Coil motion noise recorded by QUESTEM system with none shooting current at 300 meters altitude flight (Lane,1998)

Lane (1998) analyzed the characteristics of coil motion noise in time domain and Buselli (1998) analyzed the characteristics of coil motion noise in frequency domain. Based on three-component measurement, Munkholm (1997) suppressed motion-induced noise by the minimal coupling between the summation of three components' projection to an estimated direction and the motion noise[8]. Local noise prediction filtering (spies, 1988) was designed using an iterative least-squares time-domain approach predicting the vertical component of the magnetic field from two orthogonal horizontal components[9]. Lemire (1999, 2001) employed noise spline interpolation and least mean square to deal with baseline correction[10]. Davis (2006) compared movement noise to complex compound pendulum and considered not only a geometric and inductive effect in the measured signal but also altitude error from the oscillation of the towed-bird observed by both video recordings and GPS positions[11]. While Fugro Canada airborne geophysical survey company, practically adopted high pass filter or pass band filter to remove motion noise as coil motion noise covers low frequency band and improved the motion compensation and coil suspension.

Buselli (1998) shows the general spectrum of various noises and secondary field from targets in his Fig.4 at an averaging time of $0.1 \mathrm{~s}$ with the $\mathrm{Rx}$ area of $100 \mathrm{~m}^{2}$. It can be seen that the amplitude of induced voltage from motion noise is the largest one among these noises and covers the low frequency band. The amplitude of motion noise is about 200000 to $100 \mathrm{nV}$ corresponding to frequency range from $1 \mathrm{~Hz}$ to $1000 \mathrm{~Hz}$, and then the normalized induced voltage amplitudes of coil motion noise is from $2000 \mathrm{nT} / \mathrm{s}$ to $1 \mathrm{nT} / \mathrm{s}$ by dividing the $\mathrm{Rx}$ area. The amplitude of $30 \mathrm{~Hz}$ motion noise is around $10 \mathrm{nT} / \mathrm{s}$, while that of $100 \mathrm{~Hz}$ is around $3 \mathrm{nT} / \mathrm{s}$. Though the amplitude of motion noise is decreasing rapidly with the frequency increasing, it still contains frequency components higher than base frequency. So when meaarsuring noise level is over $10 \mathrm{nT} / \mathrm{s}$, high pass filter with cut-off frequency of $10 \mathrm{~Hz}$ can not remove motion noise efficiently. Munkholm (1997), Spies (1988), Davis (2006) gave motion noise reduction methods which needed extra hardware to record other field components.

This paper focuses on motion noise reduction in airborne time domain electromagnetic data using polynomial approximation. Polynomial is successfully used to approximate the baseline error (Lemire, 2001) and leveling error (Huang, 2008) in AEM data processing[12,13]. In this paper we employ polynomial to represent motion noise for each half cycle and translate polynomial coefficients problem into minimum problem via Lagrange method according to the characteristics of AEM data. Finally motion noise is subtracted from contaminant AEM data half cycle by half cycle. In the following sections, we firstly simulate a series of field data by adding a low frequency noise into the synthetic AEM data as motion noise, and then high pass filter method and polynomial approximation method are described in detail, and then are applied to the synthetic data. Motion noise reduction using polynomial approximation is free for extra hardware.

\section{Simulation of Coil Motion Noise and Noise Reduction Evaluation Indexes}

The simulation of this paper is based on an airborne time domain electromagnetic system with $4 \mathrm{~ms}$ half-sine transmitting current pulse of $25 \mathrm{~Hz}$ base frequency. According to the time domain and frequency characteristic of AEM data, pure AEM data shown in Fig.3(a) is simulated with sampling rate of $100 \mathrm{kHz}$, on time+off time data up to 2000 points each half cycle. On time data varies in cosine waveform, while synthetic data of half space $0.1 \mathrm{~S} / \mathrm{m}$ consists of off time data in exponential attenuation which is hardly to see since it is several orders of magnitude smaller than on time data. $6 \mathrm{~Hz}$ and $30 \mathrm{~Hz}$ cosine signal with different amplitudes are added to the synthetic data as coil motion noise. Fig.3(b) describes a severe motion noise on "pure" AEM data which affects off time data inversion and interpretation. Fig.4 describes signal amplitude-frequency characteristic curve before and after adding noise. It can be seen that, with low frequency motion noise increasing, obvious amplitude anomalies in $25 \mathrm{~Hz}, 6 \mathrm{~Hz}, 30 \mathrm{~Hz}$ frequency band appear and corresponding deviation of amplitudefrequency characteristic curve happen before and after adding noise.
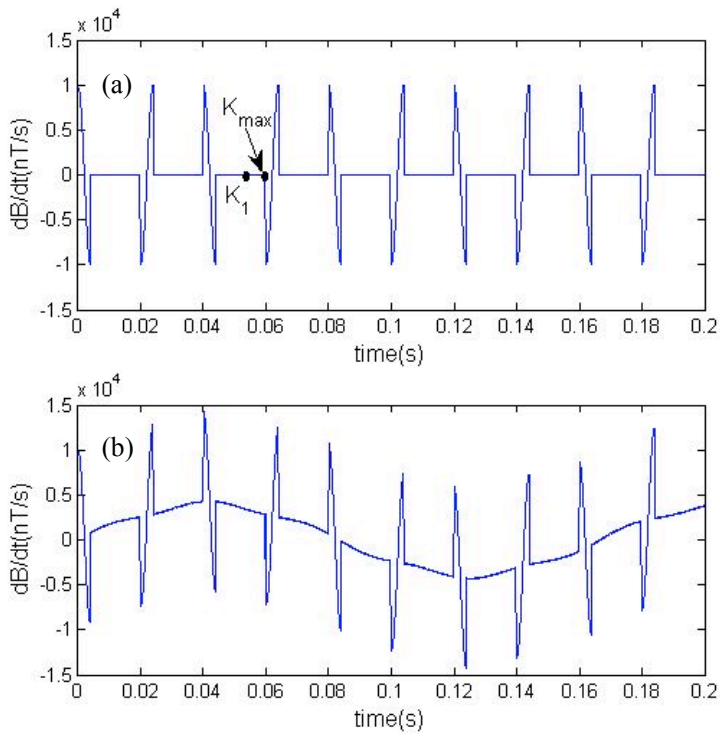

Fig. 3. Simulation of airborne time domain electromagnetic data (a) non-noise airborne time domain electromagnetic data; (b) airborne time domain electromagnetic data containing low frequency motion noise

Motion noise approximation and reduction based on polynomial is done after AEM simulation data containing motion noise known. As noise reduction evaluation indexes, 
root-mean-square error (RMSE) and signal to noise ratio (SNR) are used to inspect denoising effect.

The expression of RMSE is,

$R M S E=\left\{\left[f(n)-f^{\prime}(n)\right]^{2} / n\right\}^{1 / 2}$

Where, $f(n)$ is non-noise AEM simulation data, $f^{\prime}(n)$ is simulation data after noise reduction by polynomial approximation method. Smaller RMSE is, better noise reduction effect is.

SNR is a traditional method to judge noise magnitude in signal, the expression is,

$S N R=10 \log _{10}\left(P_{S} / P_{z}\right)$

Where, $P_{S}=\left\lfloor\sum_{n} f^{2}(n)\right\rfloor \mid n$ is signal power of non-noise AEM simulation data, $P_{z}=R M S E E^{2}$ is noise power. Bigger SNR is, better denoising effect is. According to simulation data containing motion noise in Fig.3(b), SNR is only $1.15 \mathrm{~dB}$.

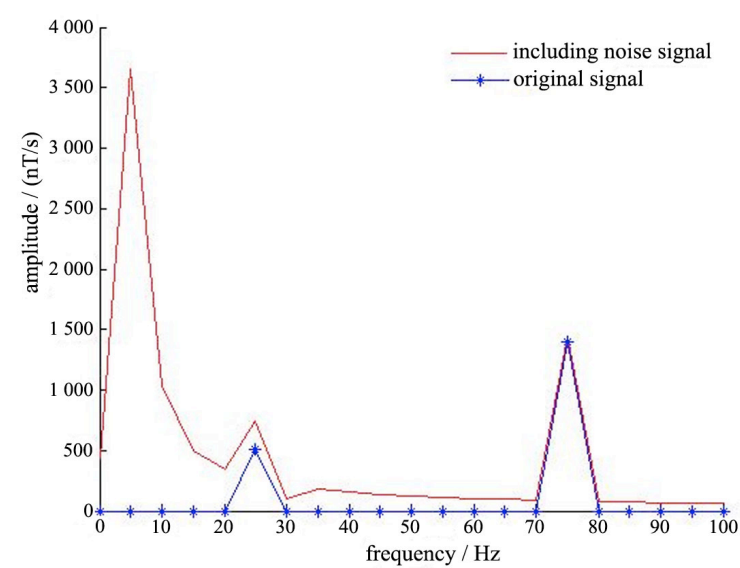

Fig.4. Amplitude and frequency characteristic curve before and after adding noise

\section{Polynomial Approximation Method and Simulation Example}

\subsection{Polynomial Approximation Method}

This method adopts a polynomial to represent coil motion noise for each half cycle constrained by the characteristics of AEM data and then subtract it from the contaminant AEM data half cycle by half cycle. For each half cycle as shown in Fig.3(a), we denote the sequence of first sample and last sample as 0 and $k_{\max }$ corresponding to sampling time 0 and $k_{\max } T_{\mathrm{S}}$ where $T_{\mathrm{S}}$ represents time interval. During off time, the secondary B field is decreased with time increasing, and when off time is relatively long (since power in the aircraft is limited, $4 \mathrm{~ms}$ pulse width of transmitting current is often designed while $25 \mathrm{~Hz}$ base frequency is chosen in China, therefore off time is $16 \mathrm{~ms}$ ), it approaches to zero or below measurement noise level when the very late time $k_{1} T_{S}$, i.e. $\left.B\right|_{t \geq k_{1} T_{S}} \approx 0$. As the measured data is the derivative of $\mathrm{B}$ field $\frac{d B}{d t}$, the B field at late time $k_{1} T_{S}$ can be derived by the integral of measured data from $0-$ to $k_{1} T_{s}$, i.e. $\left.B\right|_{t=k_{1} T_{S}}=\int_{0^{-}}^{t=k_{1}} T_{S} \frac{d B}{d t}=0 . \quad$ During the very late time after $k_{1} T_{S}$ for each half cycle the measured data are around noise level, therefore these data should be minimal in energy. Based on Lemire's analysis (1999) and above, pure AEM data of each half cycle should have the characteristics: a) the integration of AEM data should be zero from $0-$ to $k_{1} T_{s} ; \mathrm{b}$ ) there must be continuity from one half cycle to the next; c) the energy of AEM data from $k_{1} T_{S}$ to $k_{\max } T_{s}$ should be minimal.

For each half cycle, let $d_{k}$ denotes the $k$-th sample of measured data, and $f_{k}(t)$ is interpolated data between $d_{k}$ and $d_{k+1}$, while polynomial $n(t)=\sum_{j=0}^{\mathrm{N}} a_{j} t^{j}$ denotes Norder polynomial with unknown coefficients $a_{j}$ approximating motion noise at time $t, d_{p \max }$ denotes the last sample of the previous half cycle. Translate the above three characteristics into 3 equations for each half cycle:

$$
\begin{aligned}
& \left.B(t)\right|_{t=k_{1} T_{S}}=\int_{0}^{k_{1} T_{S}} \frac{d B}{d t} d t=\sum_{k=0}^{k_{1}-1} \int_{k T_{S}}^{(k+1) T_{S}}\left(f_{k}(t)-n(t)\right) d t=0 \\
& n(0)=d_{p \max } \\
& \int_{k_{1} T_{S}}^{k_{\max } T_{S}}\left(\frac{d B}{d t}\right)^{2} d t=\sum_{k=k_{1}}^{k_{\max }} \int_{k T_{S}}^{-1}(k+1) T_{S}\left(f_{k}(t)-n(t)\right)^{2} d t=\operatorname{Min}
\end{aligned}
$$

These equations can be solved by Lagrange method with the first two equations as constraints, written as

$$
L\left(a_{0}, \ldots, a_{N}, \lambda_{0}, \lambda_{1}\right)=\sum_{k=k_{1}}^{k_{\max }-1} \int_{k T_{S}}^{(k+1) T_{S}}\left(f_{k}(t)-n(t)\right)^{2} d t-\lambda_{0} l_{0}-\lambda_{1} l_{1}
$$

where $\lambda_{0}, \lambda_{1}$ are unknown Lagrange multipliers, and

$l_{0}=n(0)-d_{p \max }$

$l_{1}=\sum_{k=0}^{k-1} \int_{k}^{(k+1) T_{S}} T_{S} f_{k}(t) d t-\sum_{k=0}^{k-1} \int_{k T_{S}}^{(k+1) T_{S}} n(t) d t \quad$ are two constraint conditions. Let $L\left(a_{0}, \ldots, a_{n}, \lambda_{0}, \lambda_{1}\right)_{1}$ partial derivatives of $a_{j}$ be equal to zero, i.e. $\frac{\partial L\left(a_{0}, \ldots, a_{N}, \lambda_{0}, \lambda_{1}\right)}{\partial a_{j}}=0$ and then $\mathrm{n}+1$ equations are obtained from Eq.(4):

$$
\begin{aligned}
& 2 \underset{k=k_{1}}{k_{\max }} \int_{k T_{S}}^{(k+1) T_{S}} j^{j} n(t) d t-\lambda_{0} \delta-\lambda_{1} \sum_{k=0}^{k_{1}-1} \int_{k T_{S}}^{(k+1) T_{S}} j^{j} d t=2 \sum_{k=k_{1}}^{k_{\max }} \int_{k T_{S}}^{(k+1) T_{S}} j_{t}^{j} f_{k}(t) d t \\
& j=0,1, \ldots, \mathrm{N}
\end{aligned}
$$

where $\delta=1$ when $j=1$, otherwise $\delta=0$. 
Eq.(5) and two constraint equations are totally $\mathrm{n}+3$ equations where $\mathrm{n}+3$ unknowns are $\mathrm{n}+1$ unknowns $a_{j}$ and two unknown Lagrange multipliers $\lambda_{0}, \lambda_{1}$ which can be solved by matrix inverse programmed using MATLAB. The polynomial order is usually decided by the number of extrema of motion noise within half cycle. If there are two extrema within half cycle data, 3-order is suitable for motion noise approximation while 4 or higher order polynomials will make the code numerically unstable. Since the low frequency characteristic, half cycle of $20 \mathrm{~ms}$ often contains less than 3 extrema. Therefore, 2 or 3 order polynomial is often used.

The integral in Eq. (5) between $k T_{S}$ and $(k+1) T_{S}$ can be computed either by $\mathrm{N}$-point Gaussian integration exactly or by trapezoid integration simply when small time interval. Npoint Gaussian integration is conventionally between $[-1,1]$, say, $\int_{-1}^{1} f(t) d t=\sum_{i=1}^{N} w_{i} f\left(t_{i}\right)$ where $t_{i}$ denotes Gaussian points and $w_{i}$ Gaussian weights. For an integral over $[\mathrm{a}, \mathrm{b}]$, Gaussian integral transforms into $\int_{a}^{b} f(t) d t=\frac{b-a}{2} \sum_{i=1}^{N} w_{i} f\left(\frac{b-a}{2} t_{i}+\frac{b+a}{2}\right)$, where the value $f\left(\frac{b-a}{2} t_{i}+\frac{b+a}{2}\right)$ can be calculated by interpolation between $f(a)$ and $f(b)$. This makes all integral in Eq.(5) into summation convenient to numerical computation.

\subsection{Simulation Example Analysis}

For selecting proper polynomial order and noise entrance point, simulation research of AEM data containing motion noise is developed.

Fig.5 describes simulation results of removing motion noise to fig.3(b) with different polynomial order when $k_{1}=1800, k_{\max }=1999$, where order $\mathrm{N}$ is 2, 3, 4 for Fig.5(a), 5(b), 5(c) respectively. In order to make the result clear to the reader, especially for the off time data with small value, the following results are presented in an enlarged scale.
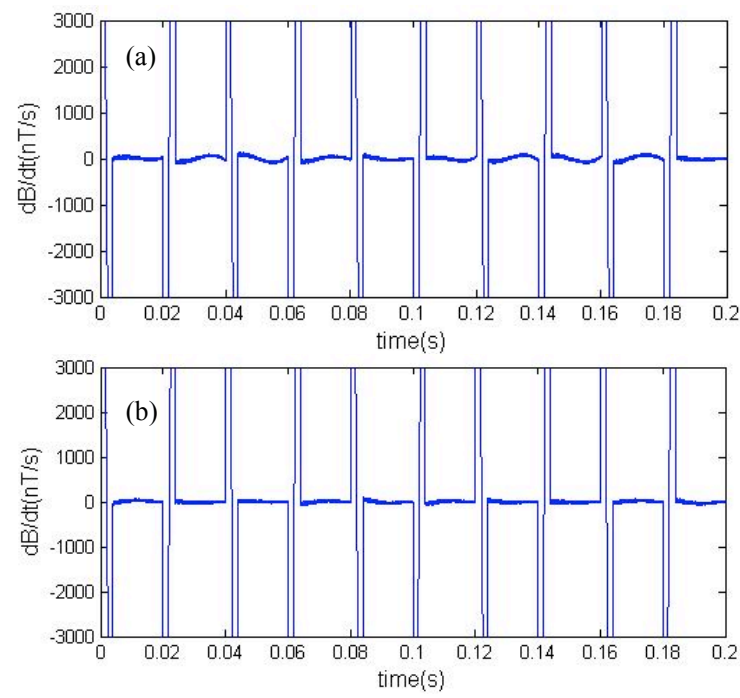

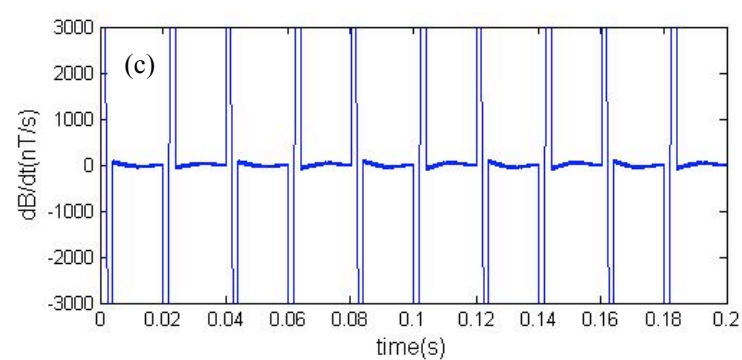

Fig.5. Simulation results of motion noise reduction with different polynomial order

\section{( $k_{1}=1800, k_{\text {max }}=1999$ ) polynomial order includes}

(a) $\mathrm{N}=2$, (b) $\mathrm{N}=3$, (c) $\mathrm{N}=4$

One can see that Fig.5(b) is the most close to the original data shown in Fig.3(a) compared with Fig.5(a) and Fig.5(c).

Table 1 gives noise reduction evaluation indexes RMSE and SNR with different polynomial order. Compared with 2 and 4 order polynomial, RMSE is minimum and SNR is maximum in 3 order polynomial, the conclusion is consistent with that of Fig.5.

Table 1 Noise reduction evaluation indexes with different polynomial order

\begin{tabular}{c|c|c}
\hline Polynomial order & $\boldsymbol{R} \boldsymbol{M S E}$ & $\boldsymbol{S N}(\mathbf{d B})$ \\
\hline 2 & 40.74 & 37.80 \\
3 & 20.12 & 43.93 \\
4 & 27.47 & 41.22 \\
\hline
\end{tabular}

It is because motion noise is relatively complex containing not only lower frequency $6 \mathrm{~Hz}$ signal but also $30 \mathrm{~Hz}$ signal higher frequency than the base frequency. This higher frequency component makes the half cycle data possibly have 2 extrama, therefore the 3 order polynomial gets the best approximation and the 2 order polynomial can not represent the characteristic of motion noise entirely. However the 4 order polynomial makes the result unstable as shown in Fig.5(c) and is often used when there are 3 extrama in half cycle data containing higher frequency.

Except for selecting proper polynomial order, noise entrance point $k_{1}$ should be confirmed. Fig.6 and table 2 describe the noise reduction simulation results with different $k_{1}$ of 3 order polynomial and corresponding noise reduction evaluation indexes. Where $k_{1}$ is 500, 1000, 1200 in fig.6(a), 6(b), 6(c) separately.

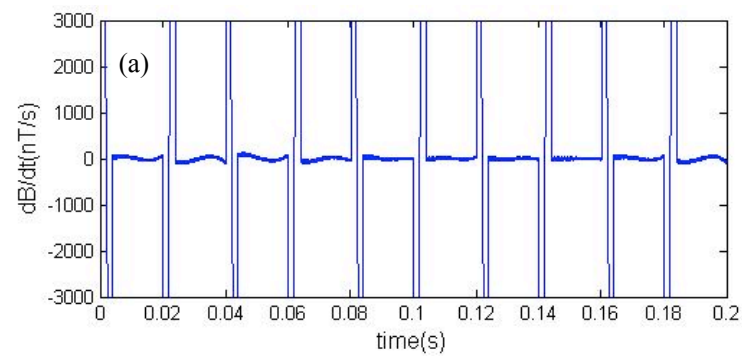



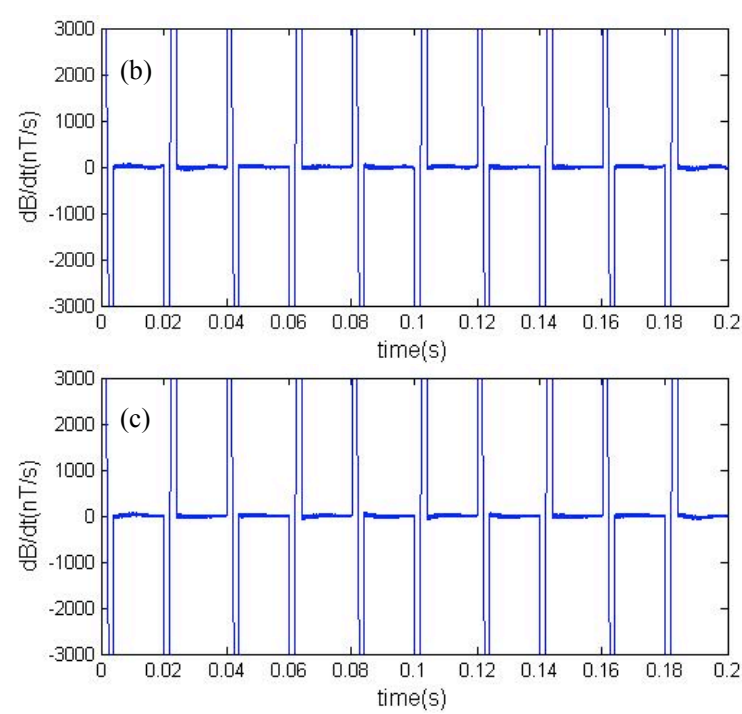

Fig.6. simulation results of motion noise reduction corresponding to 3 order polynomial with different $k_{1}$ (polynomial order $\mathrm{N}=3, k_{\max }=1999$ ).

(a) $k_{1}=500$, (b) $k_{1}=1000$, (c) $k_{1}=1200$

$\underline{\text { Table2 Noise reduction evaluation indexes with different } k_{1}}$

\begin{tabular}{c|c|c}
\hline$k_{1}$ & $\boldsymbol{R M S E}$ & $\boldsymbol{S N R}(\mathbf{d B})$ \\
\hline 500 & 32.82 & 39.68 \\
1000 & 15.51 & 46.19 \\
1200 & 14.81 & 46.58 \\
\hline
\end{tabular}

It can be seen that, because polynomial approximation is done only one time in each half cycle, noise in different time phase is different, and corresponding approximation effect is different. $k_{1}$ is the point which divides the half cycle data into 2 parts, one is effective data, the other is noise level data. When $k_{1}=500$, it means the demarcated point is at $1 \mathrm{~ms}$ after transmitting current cut off which is too early in common case. So the energy minimal problem of point 5001999 which probably contains useful signal can not get better result. With $k_{1}$ increasing gradually, RMSE decreases and SNR increases, denoising effect is continuously improved and up to best when $k_{1}=1200$. Thus, according to energy minimum principle of AEM data in noise zone, $k_{1}$ value range can be determined easily, and $k_{1}=1200$ is entrance point in noise zone. In practice, since the same flight has similar motion noise, we can choose one part of a survey line to find a better $\mathrm{N}$ and $k_{1}$ through an interactive procedure. And then these $\mathrm{N}$ and $k_{1}$ are used in other survey lines.

\subsection{Noise Neduction Capacity Comparison Between Polynomial Approximation and High Pass Filter}

Traditionally high pass filter is used to remove coil motion noise with cut off frequency at below the base frequency, e.g. around $10 \mathrm{~Hz}$. This paper designs a linear phase FIR high pass filter with $3 \mathrm{~dB}$ of $10 \mathrm{~Hz}$.

Fig.7 describes amplitude-frequency characteristic curve of denoising signal separately gained by polynomial approximation and high pass filter. One can see that, though high pass filter can filter the most of motion noise, especially effective to large amplitude $6 \mathrm{~Hz}$ low frequency noise, there is still noise residue. It is difficult for high pass filter to filter more than bass frequency noise because motion noise is composed of many different frequency components (for example $30 \mathrm{~Hz}$ in the paper). Furthermore, according to noise reduction evaluation indexes, SNR and RMSE obtained by high pass filter separately reach 22 and 286, present worse denoising effect. On the contrary, amplitude-frequency characteristic curve of denoising signal obtained by polynomial approximation is basically consistent with that of original signal, present better denoising effect.

Fig.8 describes comparing result with curve reconstruction of motion noise reduction between polynomial approximation and high pass filter. One can see that, off-time phase AEM data by polynomial denoising is basically consistent with that of non-noise AEM data, curve reconstruction effect is good. However, off-time phase AEM data by high pass filter denoising happen to deviation at late time, and only front half segment curve coincides with original decay curve, back half segment curve obviously deviates. In essence, original decay curve reflects real physical property of subsurface geologic body. Reconstruction effect of polynomial approximation is better than that of high pass filter, which further confirms the advantage of polynomial approximation method denoising.

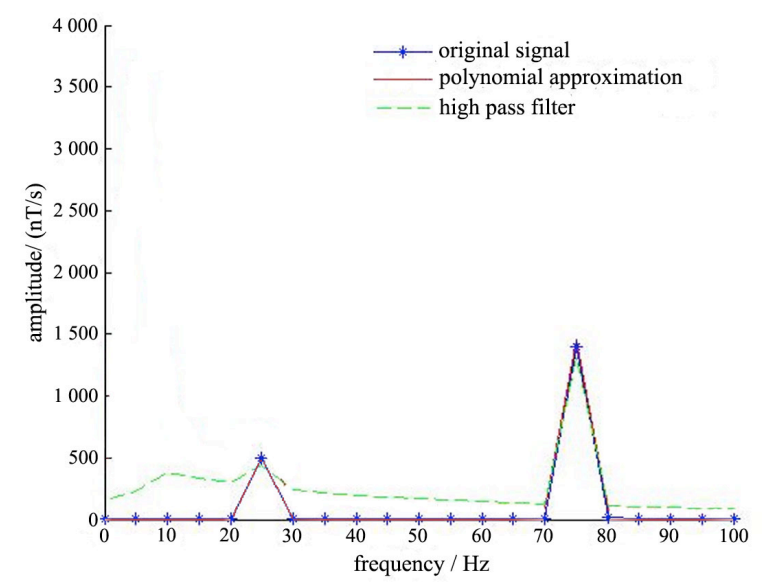

Fig.7. Comparing result with motion noise reduction of amplitude and frequency property between polynomial approximate and high pass filter

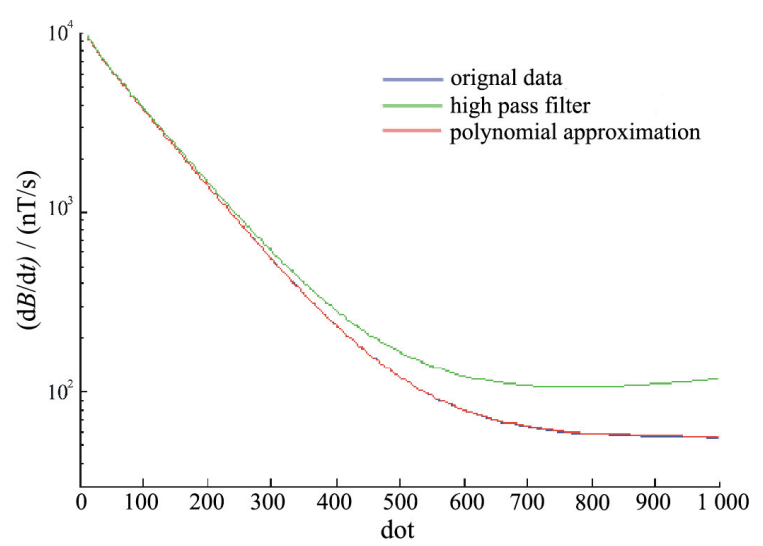

Fig.8. Comparing result with motion noise reduction of curve reconstruction between polynomial approximate and high pass filter

Here, one problem can't be neglected, because of polynomial approximation requiring whole AEM data (on 
time+off time data), actual measurement AEM data must contain on-time phase data and insure it available, therefore, application of polynomial approximation method has certain limiting condition.

\section{Conclusions}

In this paper we have investigated the polynomial approximation in removing coil motion noise in AEM data comparing with the traditional method of high pass filter. Though motion noise mainly covers low frequency band, it still has the components whose frequency band higher than base frequency. Therefore high pass filter has limitation in dealing with higher frequency motion noise. This paper, based on long off time AEM data having the following characteristics for each half cycle: a) the integral of the effective data should be zero; b) data continuity; c) the energy of the very late time data should be minimal, has employed polynomial to represent motion noise for each half cycle, and then polynomial coefficients have been solved by Lagrange method, and finally motion noise has been subtracted from the AEM data half cycle by half cycle.

The results have shown that Polynomial approximation method is an effective way to remove motion noise free for adding extra hardware with better results than traditional high pass filter.

\section{References}

1. McCracken, K.G., Oristaglio, M.L., Hohmann, G..W. Minimization of noise in electromagnetic exploration systems. Geophysics, 1986, pp. 51: 819-832.

2. Auken, E, Christiansen, A.V, Westergaard, J.H., et al. An integrated processing scheme for high-resolution airborne electromagnetic surveys, the SkyTEM system Exploration, 2009, pp. 40: 184-192.

3. Macnae, J.C, Lamontagne, Y, West, G F. Noise processing techniques for time-domain EM system. Geophysics, 1984, pp. 49: 934-948.

4. Lane R, Green A, Hu Y. Streamed data_A source of insight and improvement for time domain airborne EM. Exploration Geophysics, 1998, pp. 29: 16-23.

5. Buselli, G., Hwang, H.S. AEM noise reduction with remote referencing. Exploration Geophysics, 1998, pp. 29: 71-76.

6. Lane,R., Green, A., Golding, C, et al. An example of 3D conductivity mapping using the TEMPEST airborne electromagnetic system. Exploration Geophysics, 2000, pp. 31: $162-172$.
7. Ridsdill-Smith, T. A, Dentith, M.C. The wavelet transform in aeromagnetic processing, Geophysics, 1999, pp. 64: 1003-1013.

8. Munkholm, M.S. Motion-induced noise from vibration of a moving TEM detector coil: Characterization and suppression. Journal of applied Geophysics, 1997, pp. 36: 21-29.

9. Spies, B.R. Local noise prediction filtering for central induction transient electromagnetic sounding, Geophysics, 1988, pp. 53: 1068-1079.

10. Lemire D. Baseline asymmetry, Tau projection, B-field estimation and automatic half-cycle rejection, THEM Geophysics Inc. Technical Report, 2001.

11. Davis, A.C, Macnae, J, Robb, T. Pendulum motion in airborne HEM systems. Exploration Geophysics, 2006, pp. 37: 355-362.

12. Lemire D. Rapport sur un nouvel algorithme de correction de la ligne de base pour THEM Geophysics, Splines interpolation des moyennes etmoindres carrés (SIM), THEM Geophysics Inc. Techhnical Report, 1999.

13. Huang, H. Airborne geophysical data leveling based on line-to-line correlations, Geophysics, 2008,pp. 73: F83-F8. 